\title{
Resaerch Paper: Ten Years of Experience in Treating Patients With Digoxin Toxicity Without Using Digoxin Fab Antibody
}

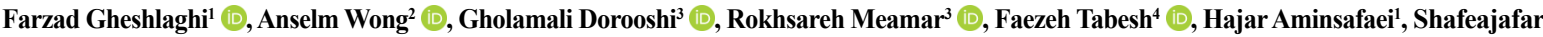
Zoofaghari' 1 , Nastaran Eizadi-Mood $^{1}$ (1)

1. Department of Clinical Toxicology, Isfahan Clinical Toxicology Research Center, School of Medicine, Isfahan University of Medical Sciences, Isfahan, Iran.

2. Victorian Poisons Information Center and Austin Toxicology Service, Austin Hospital, School of Clinical Sciences, Monash University, Victoria, Heidelberg, Australia.

3. Department of Clinical Toxicology, School of Medicine, Clinical Toxicology Research Center, Khorshid Hospital, Isfahan University of Medical Sciences, Isfahan, Iran.

4. Department of Cardiology, School of Medicine, Cardiac Rehabilitation Research Center, Cardiovascular Research Institute, Khorshid Hospital, Isfahan University of Medical Sciences, Isfahan, Iran.

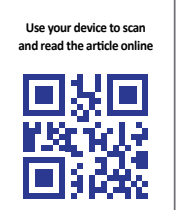

Citation: Gheshlaghi F, Wong A, Dorooshi G, Meamar R, Tabesh F, Aminsafaei H, Zoofaghari S, Eizadi-Mood N. Ten Years of Experience in Treating Patients With Digoxin Toxicity Without Using Digoxin Fab Antibody. International Journal of Medical Toxicology and Forensic Medicine. 2021; 11(1):31239. https://doi.org/10.32598/ijmtfm.v11i.31239

doi https://doi.org/10.32598/ijmtfm.v11i.31239

Article info:

Received: 5 Jul 2020

First Revision: 6 Sep 2020

Accepted: 15 Sep 2020

Published: 21 Feb 2021

\section{A B S T R A C T}

Background: Digoxin is extensively prescribed for cardiac diseases, so its chronic or acute toxicity commonly occur. Although digoxin specific antibodies (anti-digoxin Fab) are recommended to be used in patients with cardiac symptoms of digoxin toxicity, there is ongoing controversy about the effectiveness and dose of anti-digoxin Fab. Because our department lacks access to anti-digoxin Fab and the high cost of the antidote, we evaluated 10 years of experience in treating patients with digoxin toxicity without using digoxin Fab antibodies considering outcomes.

Methods: A retrospective study was performed in Khorshid Hospital, affiliated with Isfahan University of Medical Sciences, from October 2008 to September 2018. Patients with acute or chronic digoxin toxicity were included in the study. The patients' data were gathered and analyzed according to their medical documents.

Results: Out of 150 cases with digoxin toxicity, $38 \%(n=57)$ were acute and $62 \%(n=93)$ were chronic. About $64.7 \%(\mathrm{n}=97)$ were female. The most common non-cardiac manifestations of toxicity were gastrointestinal $(67.3 \%, \mathrm{n}=101)$ and neurological symptoms $(52.7 \%, \mathrm{n}=79)$. Bradyarrhythmia $(80.5 \%, \mathrm{n}=33)$ was the most cardiac manifestation in patients with acute $(15.8 \% \mathrm{n}=9)$ and chronic $(25.8 \%, \mathrm{n}=24)$ toxicity. A total of $144(96 \%)$ cases fully recovered with supportive care, and 6 patients (4\%) died. None of the cases received anti-digoxin Fab.

Conclusion: The majority of presentations with acute or chronic toxicity recovered with supportive measures without using anti-digoxin Fab.

\section{Keywords:}

Toxicity, Poisoning, Digoxin, Digoxin antibodies Fab fragments, Supportive care
* Corresponding Author:

Shafeajafar Zoofaghari, MD.

Address: Department of Clinical Toxicology, Isfahan Clinical Toxicology Research Center, School of Medicine, Isfahan University of Medical Sciences. Isfahan, Iran.

Tel: +98 (913) 1146218

E-mail: shafeajafarzoofaghari@yahoo.co 


\section{Introduction}

D

igoxin is a cardiac glycoside that is prescribed for patients with heart failure. It is also used to treat atrial flutter/fibrillation and paroxysmal atrial tachycardia [1]. In the last 20 years, the incidence of digoxin toxicity has been reported as $11 \%-30 \%$ in those taking it therapeutically [1]. Besides, the mortality rate of digoxin toxicity is reported to be $3 \%$ to $50 \%$ [2].

Digoxin specific antibodies (anti-digoxin Fab) are recommended to be used in patients who develop cardiac symptoms of digoxin toxicity; however, there is ongoing controversy about the effectiveness and dose of antidigoxin Fab [3, 4]. Anti-digoxin Fab is expensive and has a limited shelf-life, so it is difficult to keep adequate supplies if large doses are recommended. There are also adverse reactions from large anti-digoxin Fab doses such as allergic reactions, hypokalemia, rapid atrial fibrillation, and the loss of the inotropic effect of digoxin $[5,6]$.

Standard supportive therapy is the main approach for digoxin toxicity in our clinical toxicology department due to the high cost of antibody and the low rates of patients presenting with digoxin toxicity. Therefore, we evaluated 10 years of experience in treating patients with digoxin toxicity without using digoxin Fab antibodies with respect to the outcomes.

\section{Materials and Methods}

This research was a retrospective study conducted on all patients with digoxin toxicity hospitalized in Isfahan Khorshid Hospital, Isfahan City, Iran, from October 2008 to September 2018. The study was approved by the Medical Ethics Committee of Isfahan University of Medical Sciences (IR.MUI.MED.REC.1398.024).

Patients with digoxin toxicity were recruited and divided into acute (intentional or accidental) and chronic (unintentional or iatrogenic) groups. The patients' data such as age, sex, type of toxicity (acute, chronic), severity grades based on poisoning severity score (PSS), hospitalization, toxicity manifestations, serum electrolyte and digoxin levels, treatment, and outcome of the patients (recovery, death) were recorded in a checklist for each patient.

Finally, the collected data were entered into SPSS V. 22. The data were presented as Mean \pm Standard Deviation (SD) or frequency (percentage). At the level of inferential statistics, independent samples t-test and Chi- square test were used. The significance level of less than 0.05 was considered in all analyses.

\section{Results}

During the study period, 150 patients were hospitalized in Khorshid Hospital with digoxin toxicity. Out of 150 patients, 97 (64.7\%) were female, with ages ranged between 14 and 100 years (Mean \pm SD: 64.68 \pm 89.19 years). Besides, $121(80 \%)$ of the cases had chronic (unintentional or iatrogenic) toxicity. The age and type of toxicity showed significant differences between the two groups (acute or chronic toxicity) $(\mathrm{P}<0.001)$ (Table 1).

The most common symptoms in these patients were gastrointestinal $67.3 \%(\mathrm{n}=101)$ and neurological $52.6 \%$ $(\mathrm{n}=79)$ symptoms. Nausea $(91.1 \%, \mathrm{n}=92)$ and vomiting $(81.2 \%, \mathrm{n}=82)$ were the most common gastrointestinal symptoms. General weakness $(69.6 \%, \mathrm{n}=55)$ was the most common neurological symptom. Bradyarrhythmia $(80.5 \%, n=33)$ was the most common cardiovascular manifestation observed in acute and chronic digoxin toxicity. Hyperkalemia occurred in $27.9 \%(\mathrm{n}=26)$ of the chronic group and hypomagnesemia in $8.8 \%(n=5)$ of the acute group. Also, neurological symptoms and electrolytic disorders were significantly higher in chronic toxicity than acute toxicity $(\mathrm{P}<0.05)$ (Table 2 ).

The evaluation of electrolyte and metabolite levels at the times of admission and discharge between the patients with acute and chronic poisoning showed that the mean levels of potassium, sodium, and magnesium were significantly different between the two groups (acute and chronic toxicity). There was no significant difference between the two groups in the other electrolytes $(\mathrm{P}>0.05)$ (Table 3).

There was no significant difference in digoxin concentrations between patients with acute/chronic toxicity with respect to gender $(\mathrm{P}=0.097)$ and age groups $(\mathrm{P}=$ 0.243 ) at discharge (Figure 1).

Also, the patients' ECG was evaluated at the time of admission to the hospital, and every 6 hours before discharge. Results of ECG at the time of admission to the hospital and discharge are presented in Table 4. As showed in Table 4 and according to ECG results, the most common diagnosis was Atrial Fibrillation (AF) (42.7\%), which had a decreasing trend to $34.7 \%$ after treatment at discharge time (Table 4).

Treatments for these patients included atropine, pacemaker, cardioversion, and dialysis, and, if necessary, use 
Table 1. Baseline characteristics of patients with digoxin toxicity

\begin{tabular}{|c|c|c|c|c|c|}
\hline & \multirow{3}{*}{ Variables } & \multicolumn{3}{|c|}{ No. (\%) } & \multirow{3}{*}{$\mathbf{P}$} \\
\hline & & \multirow{2}{*}{ Total $(n=150)$} & \multicolumn{2}{|c|}{ Type of Toxicity } & \\
\hline & & & Acute $(n=57)$ & Chronic $(n=93)$ & \\
\hline \multirow{3}{*}{ Sex } & Female & $97(64.7)$ & $40(70.2)$ & $57(61.3)$ & \multirow{3}{*}{0.295} \\
\hline & & & & & \\
\hline & Male & $53(35.3)$ & $17(29.8)$ & $36(38.7)$ & \\
\hline \multirow{5}{*}{ Age (y) } & $<20$ & $5(3.3)$ & $5(8.8)$ & $0(0)$ & \multirow{5}{*}{$<0.001$} \\
\hline & $20-40$ & $13(8.7)$ & $13(22.8)$ & $0(0)$ & \\
\hline & $41-60$ & $22(14.7)$ & $12(21.1)$ & $10(10.8)$ & \\
\hline & $61-80$ & $62(41.3)$ & $18(31.6)$ & $44(47.3)$ & \\
\hline & $>80$ & $48(32)$ & $9(15.8)$ & $39(41.9)$ & \\
\hline \multirow{3}{*}{ Type of toxicity } & Acute (Intentional) & $22(14.7)$ & $22(38.6)$ & $0(0)$ & \multirow{3}{*}{$<0.001$} \\
\hline & Acute (Accidental) & $7(4.7)$ & $6(10.5)$ & $1(1.1)$ & \\
\hline & Chronic (Unintentional, or latrogenic) & $121(80.6)$ & $29(50.9)$ & $92(98.9)$ & \\
\hline \multicolumn{2}{|c|}{ Hospitalization; day Mean \pm SD } & $5.20 \pm 3.48$ & $3.96 \pm 3.29$ & $5.88 \pm 3.40$ & 0.001 \\
\hline \multirow{5}{*}{$\begin{array}{l}\text { Severity grades } \\
\text { at admission * }\end{array}$} & None & $21(14)$ & $16(28.1)$ & $15(16.1)$ & \multirow{5}{*}{0.715} \\
\hline & Minor & $69(46)$ & 29 (50.9\%) & $40(43)$ & \\
\hline & Moderate & $32(21.3)$ & 12 (21.1\%) & $20(21.5)$ & \\
\hline & Severe & $28(18.7)$ & 12 (21.1\%) & $16(17.2)$ & \\
\hline & Fatal & $0(0)$ & $0(0)$ & $0(0)$ & \\
\hline
\end{tabular}

*Severity grades based on Poisoning Severity Score (PSS); None (0): No symptoms or signs related to poisoning; Minor (1): Mild, transient and spontaneously resolving symptoms; Moderate (2): Pronounced or prolonged symptoms; Severe (3): Severe or life-threatening symptoms; Fatal (4): Death.

of other specialized drugs such as carvedilol, furosemide, finasteride, captopril, and atorvastatin (Table 5).

Out of 150 digoxin intoxicated patients, 144 (96\%) fully recovered. Six (4\%) patients died, of whom one patient had a cardiac arrest on admission time and 5 patients died 5 to 11 days after hospitalization. They were in the age range of 72-88 years with underlying somatic diseases, including heart failure, ischemic heart disease, hypertension, diabetic mellitus, and pulmonary thromboembolism. Digoxin level at admission in patients with full recovery group was $3.8 \mathrm{ng} / \mathrm{mL}$, in recovery group with complication 3.77 , and in the expired group 3.1 .

Detailed history and courses of cases that died were as follows:
Case 1: A 72-year-old woman, admitted to hospital with lethargy, sinus bradycardia, tachypnea, edema of extremities, and severe metabolic acidosis. The patient had a history of pulmonary emboli, myocardial infarction, and was under treatment of digoxin. Her blood level of digoxin was $2.8 \mathrm{ng} / \mathrm{dL}$ on admission. Vital signs were $\mathrm{BP}=97 / 66 \mathrm{~mm} \mathrm{Hg}, \mathrm{PR}=48$ beats $/ \mathrm{min}, \mathrm{RR}=32$ breaths $/ \mathrm{min}, \mathrm{T}=36^{\circ} \mathrm{C}$, and $\mathrm{O}_{2}$ saturation of $81 \%$. Laboratory findings showed severe metabolic acidosis: $\mathrm{K}=7.4$ $\mathrm{mEq} / \mathrm{L}$, and blood urea nitrogen/creatinine (BUN/ $\mathrm{Cr})=84 / 3.2$. The patient was a candidate for emergent hemodialysis, but she died due to respiratory arrest and arrhythmia before any intervention.

Case 2: An 88-year-old man, admitted to hospital with weakness, lethargy, sinus bradycardia, dyspnea, edema 
Table 2. Toxicity manifestations of digoxin toxicity at admission

\begin{tabular}{|c|c|c|c|c|c|}
\hline \multirow{3}{*}{\multicolumn{2}{|c|}{ Toxicity Manifestations }} & \multicolumn{3}{|c|}{ No. (\%) } & \multirow{3}{*}{$\mathbf{P}$} \\
\hline & & \multirow{2}{*}{ Total $(n=150)$} & \multicolumn{2}{|c|}{ Type of Poisoning } & \\
\hline & & & Acute $(n=57)$ & Chronic ( $n=93$ ) & \\
\hline \multicolumn{2}{|c|}{ No symptoms } & $21(14)$ & $11(19.3)$ & $10(10.7)$ & 0.154 \\
\hline \multirow{5}{*}{$\begin{array}{l}\text { Gastrointestinal } \\
\text { symptoms } \\
(67.3 \%, n=101)^{*}\end{array}$} & Nausea & $92(91.1)$ & 37 (64.9) & $55(59.1)$ & \\
\hline & Vomiting & $82(81.2)$ & $38(66.7)$ & $44(47.3)$ & \\
\hline & Stomach ache & $7(6.9)$ & $3(5.3)$ & $4(4.3)$ & 0.109 \\
\hline & Diarrhea & $9(8.9)$ & $4(7)$ & $5(5.4)$ & \\
\hline & Other & $20(19.8)$ & $11(19.3)$ & $10(10.7)$ & \\
\hline \multirow{5}{*}{$\begin{array}{c}\text { Neurological symp- } \\
\text { toms, } \\
(52.6 \%, n=79)\end{array}$} & Headache & $9(11.4)$ & $3(5.3)$ & $6(6.5)$ & \\
\hline & Dizziness & $17(21.5)$ & $6(10.5)$ & $11(11.8)$ & \\
\hline & Coma & $0(0)$ & $0(0)$ & $0(0)$ & 0.018 \\
\hline & General weakness & $55(69.6)$ & $13(22.8)$ & $42(45.2)$ & \\
\hline & Others & $12(15.2)$ & $3(5.3)$ & $9(9.7)$ & \\
\hline \multirow{4}{*}{$\begin{array}{l}\text { Cardiac symptoms, } \\
(27.3 \%, n=41)\end{array}$} & Brady arrhythmia & $33(80.5)$ & $9(15.8)$ & $24(25.8)$ & \multirow{4}{*}{0.142} \\
\hline & Tachyarrhythmia & $3(7.3)$ & $0(0)$ & $3(3.2)$ & \\
\hline & Block & $5(12.2)$ & $3(5.3)$ & $2(2.2)$ & \\
\hline & Other & $5(12.2)$ & $3(5.3)$ & $2(2.2)$ & \\
\hline \multirow{3}{*}{$\begin{array}{l}\text { Electrolyte disorder, } \\
\qquad(28.6 \%, n=43)\end{array}$} & hyperkalemia & $28(65.1)$ & $2(3.5)$ & $26(27.9)$ & \multirow{3}{*}{$<0.001$} \\
\hline & hypokalemia & $8(18.6)$ & $1(1.7)$ & $7(7.5)$ & \\
\hline & hypomagnesemia & $7(16.3)$ & $5(8.8)$ & $2(2.2)$ & \\
\hline \multirow{3}{*}{$\begin{array}{l}\text { Visual symptoms, } \\
\quad(6 \%, n=9)\end{array}$} & Photopsia & $1(11.1)$ & $1(1.7)$ & $0(0)$ & \multirow{3}{*}{0.105} \\
\hline & Reduced vision & $5(55.6)$ & $2(3.5)$ & $3(3.2)$ & \\
\hline & Visual impairment & $3(33.3)$ & $1(1.7)$ & $2(2.2)$ & \\
\hline
\end{tabular}

of lower extremities, and severe metabolic acidosis. The patient had a history of repeated pulmonary thromboembolism, myocardial infarction, and a known case of severe Congestive Heart Failure (CHF). The patient was under the treatment of digoxin, and his blood digoxin level was $3.4 \mathrm{ng} / \mathrm{dL}$ on admission, $2.59 \mathrm{ng} / \mathrm{dL}$ on the second day, and $1.65 \mathrm{ng} / \mathrm{dL}$ on the fourth day. Vital signs were $\mathrm{BP}=100 / 60 \mathrm{~mm} \mathrm{Hg}, \mathrm{PR}=49$ beats $/ \mathrm{min}, \mathrm{RR}=23$ breaths $/ \mathrm{min}, \mathrm{T}=36.5^{\circ} \mathrm{C}$, and $\mathrm{O}^{2}$ saturation of $100 \%$. The paraclinical results showed severe metabolic acidosis: $\mathrm{K}=4.8 \mathrm{mEq} / \mathrm{L}, \mathrm{BUN} / \mathrm{Cr}=22 / 1.6$. The patient was man- aged with supportive care, but he died 5 days after admission because of ventricular tachycardia.

Case 3: An 80-year-old woman, admitted to the hospital with weakness, lethargy, sinus bradycardia, tachypnea, and oliguria. The patient had a history of severe right-sided heart failure under treatment of digoxin with a serum level of $3.77 \mathrm{ng} / \mathrm{dL}$ on admission, $2.2 \mathrm{ng} / \mathrm{dL}$ on the second day, and $1.7 \mathrm{ng} / \mathrm{dL}$ on the third day. Her vital signs were $\mathrm{BP}=100 / 60 \mathrm{~mm} \mathrm{Hg}, \mathrm{PR}=49$ beats $/ \mathrm{min}$, $\mathrm{RR}=23$ breaths $/ \mathrm{min}, \mathrm{T}=36.5^{\circ} \mathrm{C}$, and $\mathrm{O}_{2}$ saturation of 
Table 3. Comparison of the average electrolytes level at the time of admission and discharge

\begin{tabular}{|c|c|c|c|c|}
\hline \multirow{2}{*}{\multicolumn{2}{|c|}{ Serum Electrolyte and Digoxin levels }} & \multicolumn{2}{|c|}{ Mean \pm SD } & \multirow{2}{*}{$\mathbf{P}$} \\
\hline & & Acute $(n=57)$ & Chronic ( $n=93$ ) & \\
\hline \multirow{2}{*}{ Sodium; mEq/L } & Admission & $136.55 \pm 17.88$ & $136.85 \pm 5.72$ & 0.883 \\
\hline & Discharge & $137.71 \pm 2.91$ & $133.73 \pm 19.00$ & 0.313 \\
\hline \multirow{2}{*}{ Potassium; $\mathrm{mEq} / \mathrm{L}$} & Admission & $4.48 \pm 0.66$ & $4.87 \pm 1.29$ & 0.045 \\
\hline & Discharge & $4.69 \pm 0.63$ & $4.25 \pm 0.81$ & $<0.001$ \\
\hline \multirow{2}{*}{ Digoxin; ng/mL } & Admission & $3.94 \pm 2.81$ & $3.74 \pm 1.69$ & 0.703 \\
\hline & Discharge & $3.43 \pm 3.15$ & $1.32 \pm 0.82$ & 0.008 \\
\hline \multirow{2}{*}{ Calcium; mg/dL } & Admission & $9.41 \pm 3.52$ & $8.88 \pm 0.85$ & $<0.001$ \\
\hline & Discharge & $10.36 \pm 5.63$ & $7.61 \pm 1.11$ & $<0.001$ \\
\hline \multirow{2}{*}{ Magnesium; $\mathrm{mEq} / \mathrm{L}$} & Admission & $3.92 \pm 2.71$ & $7.94 \pm 3.76$ & $<0.001$ \\
\hline & Discharge & $2.80 \pm 1.17$ & $3.12 \pm 1.06$ & 0.685 \\
\hline
\end{tabular}

100\%. The paraclinical results showed severe metabolic acidosis: $\mathrm{K}=3.4 \mathrm{mEq} / \mathrm{L}, \mathrm{BUN} / \mathrm{Cr}=46 / 2.2$. The patient was managed with supportive care, but she died following the recurrent and refractory to control ventricular fibrillation and tachycardia.

Case 4: A 75-year-old man was admitted to the hospital with nausea, vomiting, and anorexia. The patient was a known case of chronic obstructive pulmonary disease (COPD) and CHF and under-treatment of digoxin with a serum level of $1.88 \mathrm{ng} / \mathrm{dL}$. The patient was managed with supportive care, but he died 9 days after admission because of sepsis.

Case 5: A 79-year-old woman was admitted to the hospital with weakness, confusion, sinus bradycardia, nausea, and vomiting. The patient had a history of blood hypertension, diabetes mellitus, and severe right-sided heart failure. She was under the treatment of digoxin with a serum level of $5 \mathrm{ng} / \mathrm{dL}$ on admission, $3.2 \mathrm{ng} / \mathrm{dL}$ on the second day, and $1.4 \mathrm{ng} / \mathrm{dL}$ on the third day. Vital signs were $\mathrm{BP}=135 / 90 \mathrm{~mm} \mathrm{Hg}, \mathrm{PR}=49$ beats $/ \mathrm{min}$, $\mathrm{RR}=23$ breaths $/ \mathrm{min}, \mathrm{T}=36.5^{\circ} \mathrm{C}$, and $\mathrm{O} 2$ saturation of $100 \%$. The paraclinical results showed severe metabolic acidosis: $\mathrm{K}=6.2 \mathrm{mEq} / \mathrm{L}, \mathrm{BUN} / \mathrm{Cr}=37 / 2.6$, Aspartate transaminase $(\mathrm{AST})=322$, and Alanine transaminase $(A L T)=510$. The patient managed with supportive care and repeated hemodialysis, but she died 7 days after admission because of GI bleeding and disseminated intravascular coagulopathy.
Case 6: A 78-year-old bedridden man was admitted to the hospital with fever, tachypnea, nausea, vomiting, and anorexia. The patient was a known case of COPD and $\mathrm{CHF}$ and was under treatment of digoxin with a serum level of $1.69 \mathrm{ng} / \mathrm{dL}$. The patient was managed with supportive care and antibiotic therapy for bronchopneumonia, but he died 11 days after admission because of sepsis.

Finally, recovery in 144 patients $(96 \%)$ and death in 6 patients (4\%) were recorded. Out of 6 death cases recorded in this study, $50 \%$ was male, and $50 \%$ was female with the Mean \pm SD age of $79.67 \pm 6.22$ years; $50 \%$ of these patients had gastrointestinal, neurological, and cardiac complications simultaneously, and 4 cases $(66.7 \%)$ had electrolyte disorder, and one case $(16.7 \%)$ had vision problems. The Mean \pm SD level of digoxin in these patients was $3.11 \pm 0.83 \mathrm{ng} / \mathrm{mL}$. Besides, according to the results of age, electrolyte disorders, and type of poisoning, there were significant differences between live and dead patients $(\mathrm{P}<0.05)$ (Table 6).

\section{Discussion}

We evaluated 10 years of experience in treating patients with digoxin toxicity without using digoxin Fab antibodies with respect to the outcomes. A total of 150 cases of poisoning toxicity had been hospitalized for 10 years. In the last 20 years, the incidence of digoxin toxicity has been reported as $11 \%-30 \%$ in those taking 
Table 4. ECG diagnosis in patients with Digoxin poisoning

\begin{tabular}{|c|c|c|c|c|c|c|}
\hline \multirow{4}{*}{ ECG* } & \multicolumn{6}{|c|}{ No. (\%) } \\
\hline & \multicolumn{3}{|c|}{ Admission } & \multicolumn{3}{|c|}{ Discharge } \\
\hline & \multirow{2}{*}{ Total $(n=150)$} & \multicolumn{2}{|c|}{ Type of Poisoning } & \multirow{2}{*}{ Total $(n=150)$} & \multicolumn{2}{|c|}{ Type of Poisoning } \\
\hline & & Acute $(n=57)$ & Chronic (n=93) & & Acute $(n=57)$ & Chronic ( $n=93$ ) \\
\hline Normal & $25(16.7)$ & $18(31.6)$ & $7(7.5)$ & $27(18)$ & $19(33.3)$ & $8(8.6)$ \\
\hline $\mathrm{AF}$ & $64(42.7)$ & $15(26.3)$ & $49(52.7)$ & $52(34.7)$ & $14(24.6)$ & $38(40.9)$ \\
\hline AV Block & $20(13.2)$ & $7(12.3)$ & $11(11.8)$ & $15(10)$ & $7(12.3)$ & $8(8.6)$ \\
\hline PVCs & $2(1.3)$ & $0(0)$ & $2(2.1)$ & $1(0.7)$ & $0(0)$ & $1(1.1)$ \\
\hline SB & $10(6.7)$ & $5(8.8)$ & $5(5.4)$ & $8(5.3)$ & $4(7)$ & $4(4.3)$ \\
\hline RBBB & $1(0.7)$ & $1(1.7)$ & $0(0)$ & $2(1.3)$ & $1(1.7)$ & $1(1.1)$ \\
\hline LBBB & $17(11.3)$ & $9(15.8)$ & $8(8.6)$ & $17(11.3)$ & $9(15.8)$ & $8(8.6)$ \\
\hline LVH & $2(1.3)$ & $1(1.7)$ & $1(1.1)$ & $2(1.3)$ & $0(0)$ & $0(0)$ \\
\hline External Pacing & $0(0)$ & $0(3.5)$ & $0(0)$ & $8(5.3)$ & $0(0)$ & $8(8.6)$ \\
\hline Bigeminy & $4(2.7)$ & $1(1.7)$ & $3(3.2)$ & $3(2)$ & $0(0)$ & $3(3.2)$ \\
\hline $\mathrm{CHB}$ & $3(2)$ & $2(3.5)$ & $1(1.1)$ & $3(2)$ & $1(1.7)$ & $3(3.2)$ \\
\hline Other & 25 (16.7) & $7(12.3)$ & $18(19.3)$ & $31(20.7)$ & $10(17.9)$ & 2122.6) \\
\hline
\end{tabular}

AF: Atrial Fibrillation; AV: Atrioventricular Block; PVCs: Premature Ventricular Contractions; SB: Sinus Bradycardia; RBBB: Right Bundle Branch Block; LBBB: Left Bundle Branch Block; LVH: Left Ventricular Hypertrophy, CHB: Complete Heart Block.

*More than one diagnosis might be recorded for the patient.

it therapeutically [1]. Digoxin poisoning has decreased over recent decades [7]. This reduction is likely because of digoxin concentration monitoring and a higher awareness of physicians regarding the interactions of other drugs with digoxin.

Our results showed that patients with acute toxicity were younger than those with chronic toxicity. In this regard, young individuals are often poisoned due to suicide. But the old adults are often poisoned with drugs and unintentional poisoning. Other studies suggest that susceptibility to high digoxin concentrations increases with age $[1,2]$. Hull et al. also found that drug poisoning was 7 times higher in people over the age of 65 [8].

In our study, digoxin toxicity was more common in female than male patients. Like our study, Aarnoudse et al. reported that digoxin poisoning in women is 1.4 times more likely than in men [9]. In a study by Limon et al. (2016), $73.2 \%$ of the poisoned patients were women [10].
The major non-cardiac manifestations of digoxin poisoning included visual, digestive, and neurological disorders [10]. In this study, the most common non-cardiac symptoms were gastrointestinal symptoms (67.3\%), vomiting, and nausea with the highest frequency. This condition was followed by neurological symptoms $(57.2 \%)$, with the most prevalent manifestations of toxicity. Besides, the neurological symptoms and electrolyte disorders (such as hyperkalemia and hypokalemia) were significantly higher in chronic toxicity than in acute toxicity. Consistent with the current study, gastrointestinal symptoms, especially nausea and vomiting, have been reported as the most common clinical manifestations of digoxin toxicity in most previous studies $[10,11]$. In another study, $35 \%$ of patients had nausea and vomiting, and $27 \%$ had abdominal pain [10].

ECG findings are critical in the diagnosis and treatment of digoxin poisoning. In the previous studies, the most common ECG findings were Premature Ventricular Contractions (PVCs), Atrioventricular (AV) block, and 
Table 5. Treatment of digoxin poisoning

\begin{tabular}{|c|c|c|c|c|}
\hline \multirow{3}{*}{ Treatment* } & \multicolumn{3}{|c|}{ No. (\%) } & \multirow{3}{*}{$\mathbf{P}$} \\
\hline & \multirow{2}{*}{ Total $(n=150)$} & \multicolumn{2}{|c|}{ Type of Poisoning } & \\
\hline & & Acute $(n=57)$ & Chronic $(n=93)$ & \\
\hline Atropine & $22(14.7)$ & $10(17.5)$ & $12(12.9)$ & 0.323 \\
\hline External pace maker & $13(8.7)$ & $1(1.8)$ & 12 (12.9) & 0.016 \\
\hline Direct cardioversion & $6(4)$ & $2(3.5)$ & $4(4.3)$ & 0.773 \\
\hline Supportive measures for renal failure (dialysis) & $2(1.3)$ & $1(1.8)$ & $1(1.1)$ & 0.729 \\
\hline Other** & $49(32.6)$ & $17(11.3)$ & $32(34.4)$ & 0.347 \\
\hline
\end{tabular}

*The patients with no specific recorded treatment were under reassessment, observation, and supportive care;

${ }^{* *}$ Carvedilol, Furosemide, Finasteride, Captopril, Atorvastatin, and others.

sinus bradycardia, atrial fibrillation (AF) [11, 12], and ST-depression [10]. In our study, AF, AV block, and left bundle branch block (LBBB) were the most common findings on the ECG. The most common arrhythmias in patients with digoxin toxicity are premature ventricular contractions, which may be manifested in the form of multiple or other rhythms such as bigeminy. Arrhythmias such as junctional escape, junctional tachycardia, and bilateral ventricular tachycardia are most likely due to the onset of automated flow from a focal site due to digoxin. On the other hand, atrial flutter, PVCs, tachycardia/flutter/ventricular fibrillations are most likely due to a reentry mechanism [10].

In a study by Pita et al., the mean BUN level was significantly higher in the group of digoxin poisoned patients compared to the group of digoxin users without toxicity [11]. However, in some studies, the potassium level was significantly higher in digoxin toxicity. Hyperkalemia is not usually seen in chronic toxicity with digoxin unless a renal failure occurs simultaneously [10-13].

Out of the 150 patients with digoxin toxicity, only 6 (4\%) patients died. The mortality rate of digoxin toxicity is reported to be $3 \%$ to $50 \%$ [2]. In our study, all deaths occurred in chronic poisoning, and the causes of death were multiple organ failure. Patients were in the age range of 72-88 years with underlying somatic diseases, including heart failure, ischemic heart disease, hypertension, diabetic mellitus, and pulmonary thromboembolism. The serum digoxin concentration was lower in dead patients compared to others who survived. Previous studies have shown that patients with high serum digoxin concentrations without clinical toxicity symptoms are less at risk. The progress of digoxin toxicity symptoms is more severe in patients with elevated serum digoxin levels and clinical toxicity symptoms; these individuals are at a higher risk of death. Besides, older age, chronic toxicity, or the coincidence of other diseases, such as renal failure, can play a significant role in the mortality of these individuals [11]. In contrast, Kockova et al. (2010), in their 5-year study on patients with digoxin poisoning, showed that factors such as age, sex, the dose of digoxin, serum potassium level, and serum levels of digoxin had no significant effect on mortality due to digoxin toxicity. But the mortality rate in individuals with low ejection fraction was higher, so that left ventricular systolic function was a significant predictor of death [14].

Although digoxin specific antibodies (anti-digoxin $\mathrm{Fab}$ ) are recommended to be used in patients who developed digoxin toxicity with cardiac symptoms, there is an ongoing controversy about the effectiveness and dose of anti-digoxin Fab. The typically recommended doses aim to bind free digoxin $[3,4]$. A study showed that $1-2$ vials of anti-digoxin Fab in chronic digoxin toxicity initially bound all free digoxin confirming Fab efficacy. The time interval for a response to treatment after ending fab injection was about 19-60 minutes [15]. However, in our patients, death occurred 5 to 11 days after hospitalization.

These people had an older age than those who recovered, and all 6 had chronic poisoning. Besides, the electrolyte disorders were the most prevalent clinical manifestations in these patients, and over $50 \%$ of these patients have around two clinical manifestations of toxicity at the same time. 
Table 6. Characteristics of patients in live and expired groups in terms of digoxin poisoning

\begin{tabular}{|c|c|c|c|c|}
\hline & \multirow{2}{*}{ Characteristics } & \multicolumn{2}{|c|}{ No. (\%)/Mean \pm SD } & \multirow{2}{*}{$\mathbf{P}$} \\
\hline & & Expired $(n=6)$ & Survived ( $n=144)$ & \\
\hline \multirow{3}{*}{ Sex } & Male & $3(50)$ & $50(34.7)$ & \multirow{3}{*}{0.666} \\
\hline & & & & \\
\hline & Female & $3(50)$ & $94(65.3)$ & \\
\hline & Age $(y)$ & $79.67 \pm 6.22$ & $68.18 \pm 20.14$ & 0.003 \\
\hline \multirow{3}{*}{ Type of toxicity } & Acute (Intentional) & $0(0)$ & $22(15.3)$ & \multirow{3}{*}{0.470} \\
\hline & Acute (Accidental) & $0(0)$ & $7(4.9)$ & \\
\hline & Chronic (Unintentional, or latrogenic) & $6(100)$ & $115(79.8)$ & \\
\hline \multirow{5}{*}{$\begin{array}{l}\text { Serum Electrolyte } \\
\text { and Digoxin levels }\end{array}$} & Sodium; mEq/L & $135.67 \pm 7.87$ & $136.79 \pm 11.84$ & 0.819 \\
\hline & Potassium; mEq/L & $5.28 \pm 1.39$ & $4.71 \pm 1.11$ & 0.218 \\
\hline & Digoxin; ng/mL & $3.11 \pm 0.83$ & $3.86 \pm 2.23$ & 0.508 \\
\hline & Calcium; mg/dL & $7.88 \pm 1.66$ & $12.17 \pm 9.82$ & 0.600 \\
\hline & Magnesium; mEq/L & $7.03 \pm 9.53$ & $3.75 \pm 0.25$ & 0.860 \\
\hline \multirow{5}{*}{$\begin{array}{l}\text { Toxicity manifesta- } \\
\text { tions }\end{array}$} & Gastrointestinal & $3(50)$ & $98(68.1)$ & 0.393 \\
\hline & Neurological & $3(50)$ & $76(52.8)$ & 0.894 \\
\hline & Cardiac & $3(50)$ & $38(26.4)$ & 0.346 \\
\hline & Electrolyte disorder & $4(66.7)$ & $39(27.1)$ & 0.030 \\
\hline & Visual & $1(16.7)$ & $6(5.6)$ & 0.253 \\
\hline \multirow{6}{*}{ Treatments } & Atropine & $3(50)$ & $19(13.2)$ & 0.068 \\
\hline & External pace maker & $1(16.7)$ & $12(8.3)$ & 0.415 \\
\hline & Direct cardioversion & $4(66.7)$ & $2(1.4)$ & $<0.001$ \\
\hline & Supportive measures for renal failure(dialysis) & $0(0)$ & $2(1.4)$ & 0.755 \\
\hline & Other & $5(83.3)$ & $44(30.6)$ & 0.453 \\
\hline & Hospitalization (d) & $6.50 \pm 3.78$ & $5.14 \pm 3.47$ & 0.350 \\
\hline
\end{tabular}

Most previous studies have also stated that patients with high serum digoxin levels without clinical toxicity symptoms are less at risk. In turn, the progress of digoxin toxicity symptoms is worse in patients with elevated serum digoxin levels and clinical toxicity symptoms; these individuals are at higher risk of death [16]. Besides, older age, chronic toxicity, or the coincidence of other diseases, such as renal failure, can play a significant role in the mortality of these individuals $[10,17]$. In contrast, Kockova et al., in their 5-year study on patients with digoxin poisoning, found that factors such as age, sex, the dose of digoxin, serum potassium level, and serum levels of digoxin had no significant effect on mortality due to digoxin toxicity [14].

Because of no access to Fab in Iran, treatments for these patients include atropine, external pacemaker, direct cardioversion, and supportive measures for renal failure (dialysis) if necessary, and use of other drugs such as carvedilol, furosemide.

Although the studies with access to digoxin antibody (DigiFab) and its prescription also indicated that using 

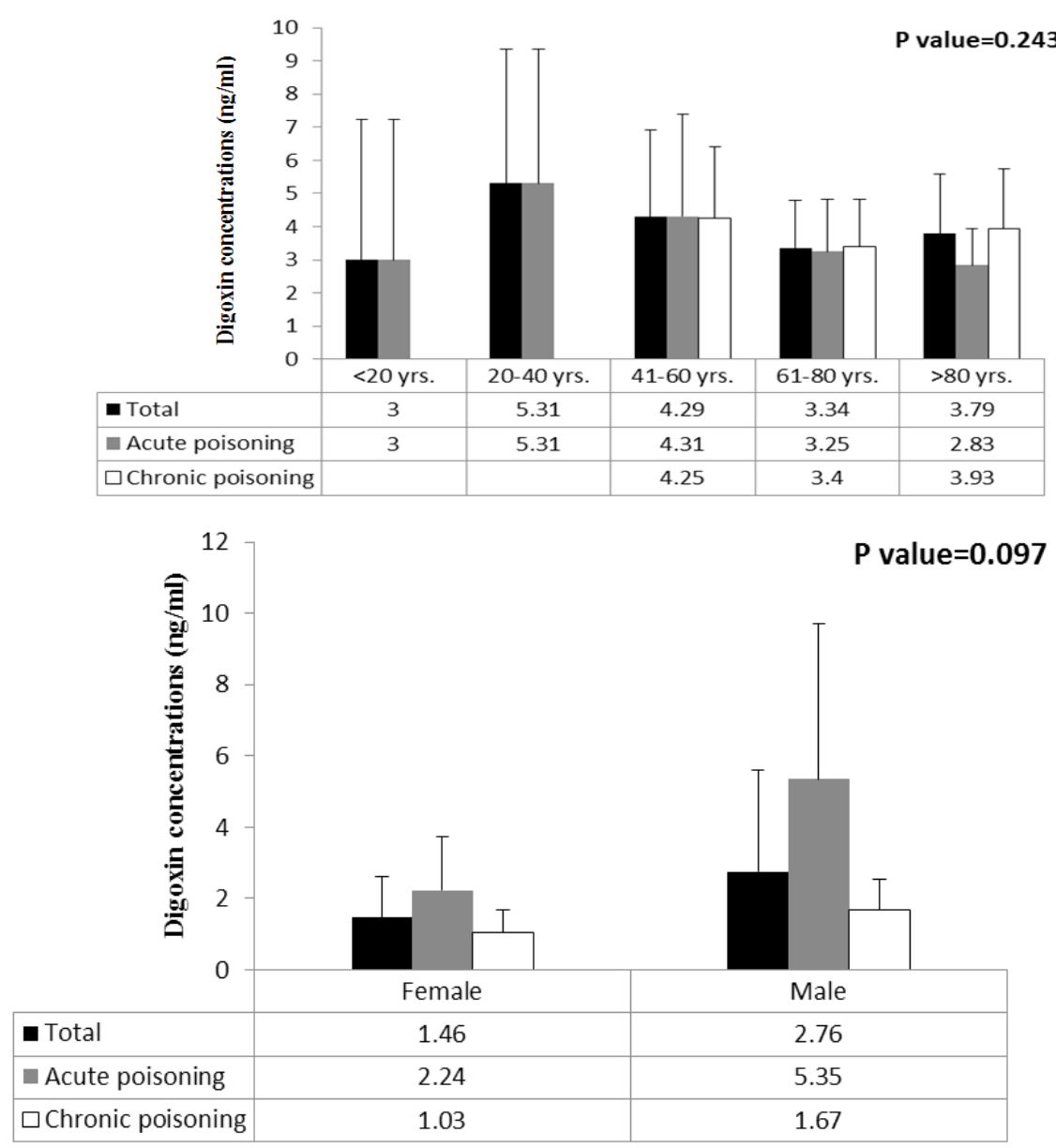

Figure 1. Comparison of mean digoxin concentration by sex at discharge

International Journal of
Medical Toxicology \& Forensic Medicine

it, over $80 \%$ of the toxicity symptoms in these patients were eliminated, $10 \%$ showed complete recovery, and $10 \%$ showed no response to treatment $[13,18]$. Therefore, it can be stated that there is no difference between them and the current study in terms of success. However, the only difference between these studies compared to our work regarding the use of DigiFab is quick treatment and response. In their studies, the time for response to therapy after ending Fab injection was about 19-60 minutes, while the hospitalization duration in our study was 1-8 days. Although this duration is long compared to other studies, given the existing economic conditions and constraints of the developing countries, especially in Iran, finding alternative medical methods with the best efficacy in these patients seems necessary and vital.

\section{Conclusion}

In the absence of digoxin Fab antibodies to manage digoxin toxicity, supportive measures with chronotropes/ inotropes, electrolyte correction, and organ support are sufficient in treating acute or chronic digoxin toxicity.

\section{Declaration of patient consent}

The authors certify that they have obtained all appropriate patient consent forms. The patients gave their consent for their images and other clinical information to be reported in the journal. The patients understand that their names and initials will not be published, and due efforts will be made to conceal their identity, but anonymity cannot be guaranteed.

\section{Ethical Considerations}

\section{Compliance with ethical guidelines}

The present study was approved by the Ethics Committee of Isfahan University of Medical Sciences (28 April 2019, IR.MUI.MED.REC.1399.024).

\section{Funding}

This research did not receive any grant from funding agencies in the public, commercial, or non-profit sectors. 


\section{Author's contributions}

Conceptualization and Methodology: Farzad Gheshlaghi, Shafeajafar Zoofaghari, and Nastaran EizadiMood; Data collection: Shafeajafar Zoofaghari and Hajar Aminsafaei; Data analysis: Farzad Gheshlaghi, Shafeajafar Zoofaghari, Nastaran Eizadi-Mood, and Anselm Wong; Writing - original draft: Farzad Gheshlaghi, Shafeajafar Zoofaghari, Nastaran Eizadi-Mood, Anselm Wong, and SG; Supervision: All authors.

\section{Conflict of interest}

The authors declared no conflict of interest.

\section{References}

[1] Steiner JF, Robbins LJ, Hammermeister KE, Roth SC, Hammond WS. Incidence of digoxin toxicity in outpatients. The West J Med. 1994; 161(5):474-8. [PMCID] [PMID]

[2] Pincus M. Management of digoxin toxicity. Aust Prescr. 2016; 39(1):18-20. [DOI:10.18773/austprescr.2016.006] [PMID] [PMCID]

[3] Bateman DN. Digoxin-specific antibody fragments: How much and when? Toxicol Rev. 2004; 23(3):135-43. [DOI:10.2165/00139709-200423030-00001] [PMID]

[4] Lapostolle F, Borron SW, Verdier C, Arnaud F, Couvreur J, Megarbane B, et al. Assessment of digoxin antibody use in patients with elevated serum digoxin following chronic or acute exposure. Intensive Care Med. 2008; 34(8):1448-53. [DOI:10.1007/s00134-008-1092-x] [PMID]

[5] Kirkpatrick $\mathrm{CH}$. Allergic histories and reactions of patients treated with digoxin immune Fab (ovine) antibody. The digibind study advisory panel. Am J Emerg Med. 1991; 9(2 Suppl 1):7-10. [DOI:10.1016/0735-6757(91)90160-L]

[6] Flanagan RJ, Jones AL. Fab antibody fragments: Some applications in clinical toxicology. Drug safety. 2004; 27(14):111533. [DOI:10.2165/00002018-200427140-00004] [PMID]

[7] Chan BS, Buckley NA. Digoxin-specific antibody fragments in the treatment of digoxin toxicity. Clin Toxicol (Phila). 2014; 52(8):824-36. [DOI:10.3109/15563650.2014.943907] [PMID]

[8] Hull SM, Mackintosh A. Discontinuation of maintenance digoxin therapy in general practice. Lancet (London, England). 1977; 2(8047):1054-5. [DOI:10.1016/S0140-6736(77)91886-4]

[9] Aarnoudse AL, Dieleman JP, Stricker BH. Age-and genderspecific incidence of hospitalisation for digoxin intoxication. Drug Saf. 2007; 30(5):431-6. [DOI:10.2165/00002018200730050-00006] [PMID]

[10] Limon G, Ersoy G, Oray NC, Bayram B, Limon O. Retrospective evaluation of patients with elevated digoxin levels at an emergency department. Turk J Emerg Med. 2016; 16(1):1721. [DOI:10.1016/j.tjem.2015.10.001] [PMID] [PMCID]
[11] Pita-Fernandez S, Lombardia-Cortina M, Orozco-Veltran D, Gil-Guillen V. Clinical manifestations of elderly patients with digitalis intoxication in the emergency department. Arch Gerontol Geriatr. 2011; 53(2):e106-10. [DOI:10.1016/j.archger.2010.07.003] [PMID]

[12] Kirilmaz B, Saygi S, Gungor H, Onsel Turk U, Alioglu E Akyuz S, et al. Digoxin intoxication: An old enemy in modern era. J Geriatr Cardiol. 2012; 9(3):237-42. [DOI:10.3724/ SP.J.1263.2012.01101] [PMID] [PMCID]

[13] Miura T, Kojima R, Sugiura Y, Mizutani M, Takatsu F, Suzuki Y. Effect of aging on the incidence of digoxin toxicity. Ann Pharmacother. 2000; 34(4):427-32. [DOI:10.1345/ aph.19103] [PMID]

[14] Santoro F, Ieva R, Ferraretti A, Carpagnano G, Lodispoto $\mathrm{M}$, De Gennaro L, et al. Acute renal failure, digoxin toxicity and brady-arrhythmia as possible triggers in Tako-Tsubo cardiomyopathy. Int J Cardiol. 2013; 165(3):e51-2. [DOI:10.1016/j. ijcard.2012.11.015] [PMID]

[15] Kockova R, Skvaril J, Cernohous M, Maly M, Kocka V, Linhart A. Five year two center retrospective analysis of patients with toxic digoxin serum concentration. Int J Cardiol. 2011; 146(3):447-8. [DOI:10.1016/j.ijcard.2010.10.114] [PMID]

[16] Chan BS, Isbister GK, O'Leary M, Chiew A, Buckley NA Efficacy and effectiveness of anti-digoxin antibodies in chronic digoxin poisonings from the DORA study (ATOM-1). Clin Toxicol (Phila). 2016; 54(6):488-94. [DOI:10.1080/15563650.20 16.1175620] [PMID]

[17] Goldberger ZD, Goldberger AL. Therapeutic ranges of serum digoxin concentrations in patients with heart failure. Am J Cardiol. 2012; 109(12):1818-21. [DOI:10.1016/j.amjcard.2012.02.028] [PMID] [PMCID]

[18] Chan KE, Lazarus JM, Hakim RM. Digoxin associates with mortality in ESRD. J Am Soc Nephrol. 2010; 21(9):1550-9. [DOI:10.1681/ASN.2009101047] [PMID] [PMCID] 